\title{
Norois
}

Environnement, aménagement, société

$240 \mid 2016$

Aménagement foncier, risque côtier, évolution des fleuves

\section{La dynamique du paysage et de l'occupation humaine entre Loire et Cisse}

Landscape evolution and human settlement between Loire and Cisse

\section{Pauline Thonniet et Jean-Baptiste Rigot}

\section{OpenEdition}

Journals

Édition électronique

URL : https://journals.openedition.org/norois/5948

DOI : $10.4000 /$ norois. 5948

ISBN : 978-2-7535-5362-0

ISSN : 1760-8546

Éditeur

Presses universitaires de Rennes

Édition imprimée

Date de publication : 30 novembre 2016

Pagination : $59-76$

ISBN : 978-2-7535-5356-9

ISSN : 0029-182X

Référence électronique

Pauline Thonniet et Jean-Baptiste Rigot, «La dynamique du paysage et de l'occupation humaine entre Loire et Cisse », Norois [En ligne], 240 | 2016, mis en ligne le 30 novembre 2018, consulté le 13 janvier 2022. URL : http://journals.openedition.org/norois/5948; DOI : https://doi.org/10.4000/norois.5948 


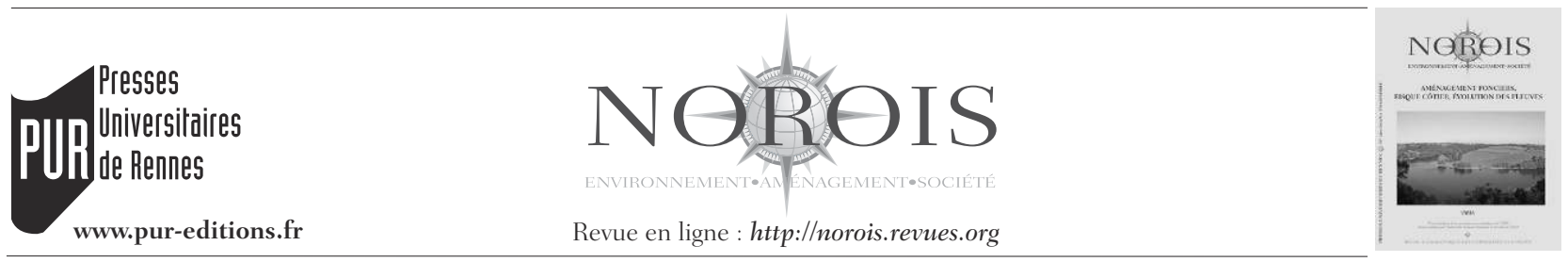

\title{
La dynamique du paysage et de l'occupation humaine entre Loire et Cisse ${ }^{1}$
}

\author{
Landscape Evolution and Human Settlement between Loire River and Cisse River
}

\author{
Pauline Thonniet ${ }^{\mathrm{a}}$, Jean-Baptiste Rigot ${ }^{\mathrm{a}}$
}

* auteur correspondant (jb.rigot@univ-tours.fr)
a UMR 7324 CITERES, Équipe Archéologie et Territoires. MSH Val de Loire-33 allée Ferdinand-de-Lesseps,
BP 60449, 37204 Touns Cedex 03. (pauline.thonniet@etu.univ-tours.fr)

Résumé : Cet article s'attache à présenter les premiers résultats d'une étude sur la dynamique des paysages et de l'occupation humaine sur le temps long dans une portion de la plaine alluviale de la Loire. Le secteur choisi pour cette recherche est le val de Cisse. Ce type de milieu présente l'intérêt de regrouper un certain nombre de problématiques liées à l'occupation de la vallée : insertion des riverains et mise en place des activités économiques dans un espace sensible, prise en compte des conditions hydriques, aménagements, exposition au risque d'inondation. Par ailleurs, il s'agit d'un espace marqueur de l'évolution du milieu. L'analyse des documents d'archives, des données archéologiques, des données cartographiques et des données géomorphologiques, dans une perspective géoarchéologique, fournit une première approche des modalités d'occupation et d'exploitation du sol depuis le Néolithique. L'occupation ancienne, pérenne, mais lâche et concentrée dans des secteurs protégés, témoigne d'une appropriation de l'espace essentiellement centrée sur la mise en valeur agricole. Dans la partie aval du val en particulier, l'absence de bourg confirme la déconnexion entre l'espace exploité et les lieux de l'occupation humaine et suggère la présence ancienne d'une zone d'expansion des crues de la Loire.

\begin{abstract}
This article presents the first results of a study on the dynamics of the landscape settlement over the long term in a portion of the allwial plain of the Loire. The area chosen is the Cisse Valley. This environment combines a certain number of problems connected to the occupation of the Loire Valley: the integration of the local population and the implementation of economic activities in a sensitive area, hydromorphic conditions, land development, and flood risk. Furthermore this space is a marker for local river dynamics and environmental changes. The analysis of the documentary sources, archaeological data, cartographic data and geomorphological data, from a geoarchaeological perspective, provides an initial approach to understanding how the land has been occupied and exploited since since the Neolithic. The ancient, long-standing but uneven occupation of this area, concentrated in protected sectors, testifies to an appropriation of the space based upon agricultural development. The absence of a village in the downstream part of the valley confirms a separation between inhabited space and exploited space, suggesting the presence of an ancient flood plain of the Loire.
\end{abstract}

Mots clé : Géoarchéologie - Loire moyenne - paysage fluvial - occupation du sol - mise en valeur - Holocène

Keywords: Geoarchaeology - River Loire - fluvial landscape - land use - agricultural development - Holocene

1. Article initialement programmé dans le dossier «Diversité des paysages fluviaux », Norois, n² 237, 2015/4. 


\section{INTRODUCTION}

Depuis les années 1980 et les travaux de Bravard et al. (1987, 1989), une approche interdisciplinaire de l'étude des corridors fluviaux sur le temps long a vu le jour en France, animée en particulier par les géographes et les archéologues. Cette perspective, identifiée sous le terme de géoarchéologie fluviale, interroge la transformation des vallées et les modalités d'occupation du sol dans ces milieux d'un point de vu dynamique, à la fois dans l'espace et dans le temps. Sans être exhaustifs, on peut citer un certain nombre de publications récentes qui témoignent de la vigueur de cette approche : Bravard (2004), Carcaud (2004), Alline (2005), Petit (dir.) (2005), Salvador (2005), Arnaud-Fassetta (2007), Morhange et Provansal (2007), Castanet (2008), Arnaud-Fassetta et Carcaud (dir.) (2015). Ces travaux s'appuient et participent des recherches menées en géomorphologie fluviales depuis les travaux novateurs de S.A. Schumm (1977). Citons les synthèses de Knighton (1984), Amoros et Petts (1993) ou encore Bravard et Petit (1997). Enfin, le travail mené actuellement sur la Cisse s'adosse à un cadre problématique plus large portant sur l'anthropisation des fonds de vallée. On peut notamment citer la somme que constitue les publications du programme Archaeomedes et en particulier l'ouvrage de Van der Leeuw et al. (2003).

C'est dans ce cadre scientifique que le travail présenté ici a été réalisé. Il est fondé sur l'étude d'un tronçon de la vallée de la Loire moyenne étiré de Chouzy-sur-Cisse, en aval de Blois, à Vouvray, quelques kilomètres en amont de Tours (figures 1 et 2). Cet espace présente une physionomie particulière caractérisée par deux unités morphologiques bien identifiées, longeant le lit endigué : une dépression latérale à la Loire empruntée par l'actuelle rivière Cisse et un bourrelet de rive surplombant le chenal actif (figure 2). Cet « épanouissement de la plaine alluviale » (Carcaud, 2004) porte le nom de val. Il supporte une occupation humaine ancienne mais lâche. L'originalité de ce secteur, inséré dans la plaine alluviale de la Loire, a conduit à la mise en place d'une recherche fondée sur le croisement de données humaines et naturelles sur le temps long. Les objectifs poursuivis visent en particulier à mieux comprendre l'insertion des populations dans le val à travers la mise en valeur de cet espace humide, au regard des conditions de vie et du risque d'inondation. Dans cette perspective, nous empruntons plus directement aux recherches réalisées sur le bassin versant de la Loire depuis les travaux fondateurs de Roger Dion (Dion, 1934 et 1961), tant au plan des dynamiques paysagères que du peuplement (voir par exemple Carcaud et Garcin, 2001 ; Carcaud et al., 2002 ; Burnouf et al., 2003ab; Carcaud, 2004; Castanet, 2008; Grivel, 2008; Morin, 2011, Arthuis et Monteil (dir.) 2015). L'objectif de cet article est de fournir une synthèse des premiers résultats centrés sur la dynamique du paysage. Deux approches sont croisés : l'évolution du milieu à l'Holocène et la dynamique du peuplement depuis le Néolithique.

Dans un premier temps nous proposons un scénario de mise en place du val à l'Holocène. À la suite des travaux du PCR «Géoarchéologie de la

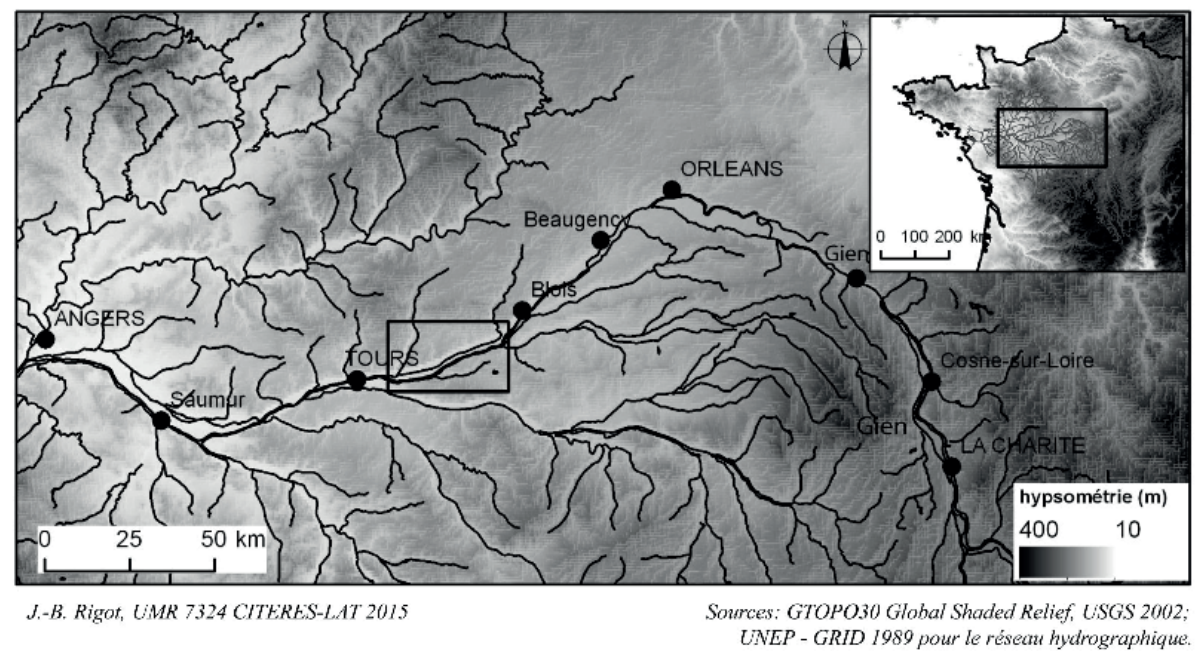

Figure 1 : Loire moyenne. Réseau hydrographique du bassin de la Loire, principales agglomérations et zone d'étude

Middle Loire Valley. Hydrographic network of the Loire basin, main built-up areas, and study area 
Loire Moyenne et de ses Marges », (Carcaud et Garcin, 2001), Nathalie Carcaud (2004) a proposé un modèle d'évolution de la mosaïque fluviale des vals en Loire Moyenne que l'on peut résumer en quelques points fondamentaux : une phase de tressage à la charnière Pléniglaciaire-Tardiglaciaire; la rétraction de l'écoulement à un chenal unique à la transition Tardiglaciaire-Holocène, avec la mise en place progressive des unités morphologiques des vals; un comblement progressif des bras secondaires encore actif durant l'Holocène et en particulier au Préboréal; Enfin, des réactivations périodiques de chenaux secondaires dans le val, en raison de péjorations climatiques secondaires au Néolithique Moyen et à l'époque gallo-romaine.

Dès lors, nous souhaitons interroger ce modèle afin de voir s'il peut être appliqué à notre secteur. À partir d'une étude géomorphologique, sont mis en évidence les modalités d'édification du bourrelet de rive, de la dépression latérale occupée aujourd'hui par la Cisse et en parallèle, de la fixation du chenal de la Loire à sa position actuelle. Cette première étape permet la mise en place d'un cadre dans lequel les questions concernant la dynamique du peuplement peuvent être développées. C'est ainsi que l'analyse du parcellaire vient conforter des observations faites avec les analyses topographiques et géomorphologiques. Le croisement de ces sources montre combien les héritages humains sont étroitement conditionnés, dans ce secteur, par ceux de la nature.

Dans un second temps, on présente les dynamiques d'occupation du sol dans le val de Cisse. Un des points développé, bien représentatif de l'appropriation de l'espace, est la « construction » de la rivière Cisse qui occupe la dépression latérale. Un certain nombre d'indices indiquent en effet qu'on a là clairement une "fabrication » des sociétés riveraines (Barraud, 2009). La rivière constitue donc un enjeu majeur dans ce secteur que notre étude vise à mieux cerner.

La répartition de l'occupation est aussi au centre de notre réflexion, tant elle est particulière : en effet, toute la partie aval du val, après Amboise, sur laquelle nous nous sommes concentrés dans le cadre de ce travail, n'est que sporadiquement occupée. On ne compte aujourd'hui que quelques hameaux et une seule commune (Négron) dont le bourg est situé dans la périphérie d'Amboise, sur le bourrelet de rive. Par ailleurs d'après les cartes actuelles et en remontant à la carte de Cassini, on n'observe qu'une seule autre commune dont le bourg est situé sur le bourrelet de rive, en amont d'Amboise (Veuves) (figure 2A). Cette répartition est originale. Elle ne s'observe pas dans le Val d'Authion, sur le bourrelet duquel s'égrènent de nombreux bourgs, ni même dans le «val triple » (Carcaud, 2004) en aval de Tours, où les îles de Berthenay et de Bréhémont sont marquées par la présence de 4 bourgs. Cela vient apparemment contredire l'intense aménagement agricole qui est une caractéristique du val de Cisse et interroge l'organisation spatiale sur le temps long. Enfin, sans l'aborder directement, nous verrons comment le risque d'inondation éclaire plusieurs questions soulevées par la répartition des populations dans le Val de Cisse.

\section{ZoNe D'ÉTUdE, DONNÉES ET MÉTHODES}

\section{Cadre géographique du val de Cisse}

\section{Contexte géologique et données hydrologiques}

Le val de Cisse appartient au cours moyen de la Loire situé au sud du Bassin Parisien (figure 1). Dans ce bassin sédimentaire, le fleuve s'est constitué un large espace de circulation au dépend de formations alluviales récentes. L'ensemble est incisé dans les calcaires de Beauce (Tertiaire) puis, à l'aval de Blois, dans les calcaires du Crétacé supérieur (Turonien et Sénonien). Longeant les coteaux taillés dans le Sénonien en rive gauche, la Loire du val de Cisse est relativement rectiligne, ponctuée régulièrement d'îles et possédant quelques bras secondaires. En termes de style fluvial, ce tronçon n'appartient pas à une classification particulière. Le lit mineur, enserré dans des digues insubmersibles (les levées) détermine une Loire " corsetée », rectiligne et parsemée d'île (Bazin et Gautier, 1996).

Le climat en Loire moyenne est dominé par les influences océaniques. Ainsi, la Loire connaît en général un régime de hautes eaux en hiver et secondairement au printemps et des basses eaux en été. Le débit moyen annuel à l'aval immédiat du val de Cisse (station de Tours) est de $356 \mathrm{~m}^{3}$ sur les 60 dernières années [www.hydro.eaufrance.fr]. Les débits hivernaux atteignant $616 \mathrm{~m}^{3}$ en moyenne en février 


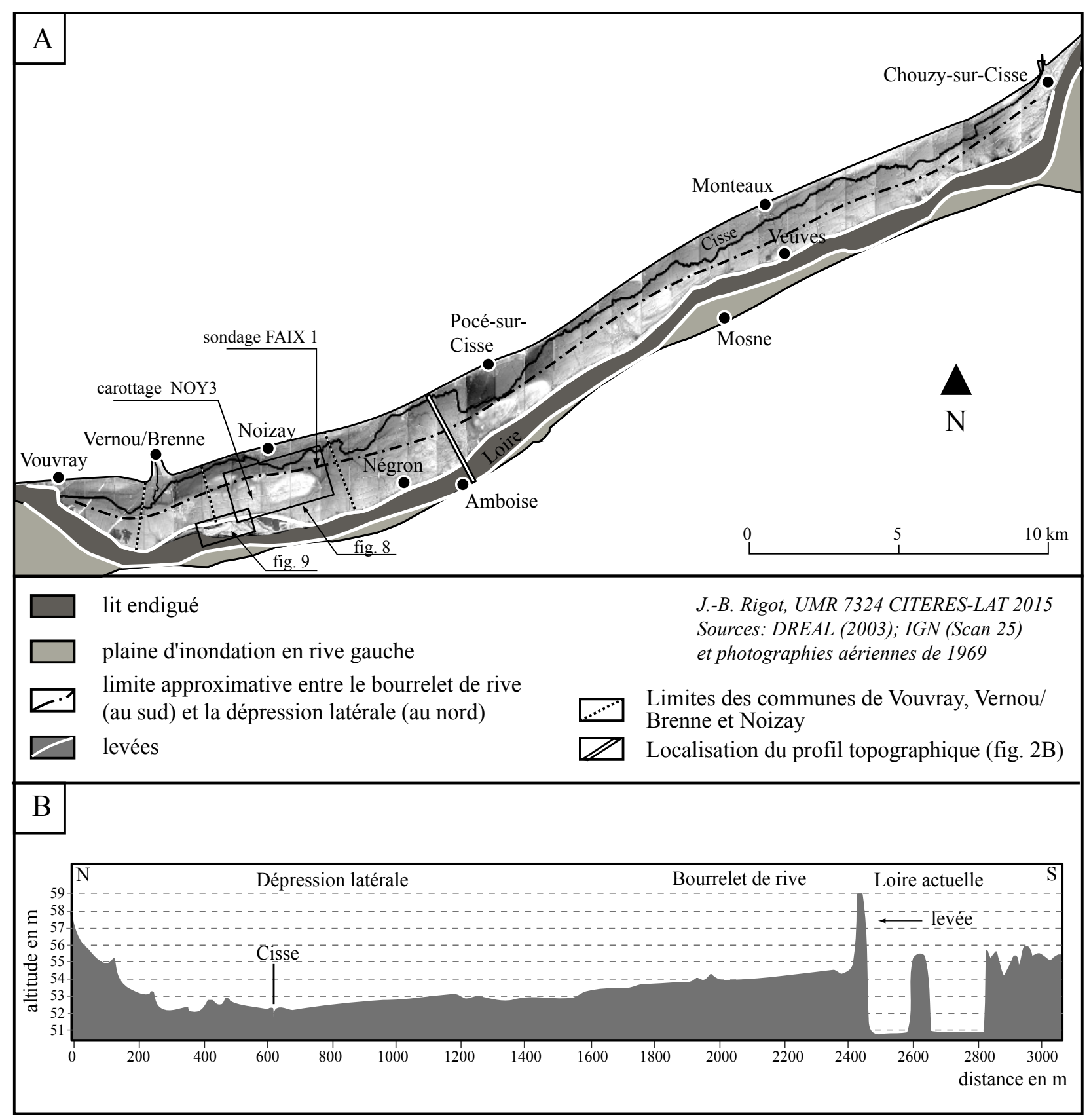

Figure 2 : A : unités morphologiques du val de Cisse; B : profil transversal du val de Cisse à la hauteur d'Amboise, d'après des données LiDAR (DREAL, 2003)

A: Morphological units of the Cisse Valley; B: Cross section of the Cisse Valley at Amboise, based on LiDAR data (DREAL, 2003)

sur la même période tandis que les débits estivaux descendent à $114 \mathrm{~m}^{3}$ en août. Par ailleurs le cours d'eau est caractérisé par d'importants contrastes de débits entre les crues et les réssuyages rapides. Ainsi les événements hydrologiques de 2007 montrent des débits moyens journaliers atteignant $1600 \mathrm{~m}^{3}$ au début du mois de mars pour redescendre assez rapidement à 500 puis $400 \mathrm{~m}^{3}$ à la fin de ce même mois. Ce contexte hydrologique détermine un cours d'eau parfois imprévisible voire dangereux pour ses riverains. 


\section{Secteur d'étude}

L'espace concerné par cette étude est une portion de la plaine alluviale de la Loire s'étendant entre Chouzy-sur-Cisse en amont et Vouvray en aval, quelques kilomètres en amont de Tours (figure 2). Il se caractérise par une large bande de terre étirée sur plus de $35 \mathrm{~km}$. Celle-ci occupe l'essentiel du fond de vallée dans cette portion du fleuve, soit environ $75 \%$ de la surface, le reste étant occupé par le chenal actif de la Loire.

Cette morphologie n'est pas unique en Loire moyenne et a déjà fait l'objet d'études. Citons en particulier les travaux de N. Carcaud sur le val d'Authion ${ }^{2}$ aux environs de Bourgueil, ou ceux de Garcin et al. $(1999,2001)$ sur le val d'Avaray en amont de Blois.

Deux unités morphologiques s'y succèdent parallèlement : une dépression allongée, latérale au fleuve, occupée par un petit affluent de la Loire (ici, la Cisse) et un bourrelet de rive bordant le chenal (figure 2). L'ensemble forme un paysage au modelé apparemment peu marqué. Cependant, après étude des photographies aériennes et des données LiDAR (DREAL, 2003), on constate que les deux unités morphologiques ressortent très nettement dans la topographie. Jusqu'à 3 mètres de dénivelée les séparent (figure 2B). Des bombements allongés de quelques centaines de mètres façonnent parfois le bourrelet, qui dès lors domine le fond de la dépression de plus de 4 mètres (figure 3A-B). La Loire, quant à elle, coule en général à 1 ou 2 mètres en contrebas de la dépression. Si elle bénéficiait originellement d'un vaste espace de circulation, elle est aujourd'hui et depuis le début de l'époque moderne, corsetée entre deux hautes digues qui réduisent considérablement l'étendue possible de son épanouissement (figures 2 et 3 ).

\section{Sources et méthodes}

\section{Sources planimétriques et textuelles}

Les sources planimétriques (tableau 1) sont issues des archives départementales d'Indre et Loire (ADIL) et des archives nationales (AN). La docu-

2. Voir notamment la synthèse de ses différents travaux sur la Loire dans la cadre de son habilitation à diriger des recherches : Carcaud N., 2004. D'espace et de temps : un itinéraire de recherche et d'enseignement sur les anthroposystemes fluviaux, mémoire d'HDR, université d'Angers, 216 p. mentation est peu abondante (effet de source lié à la conservation des documents ou bien à l'archivage des données). Les plans, dont le plus ancien est daté de 1733, concernent principalement la Loire. Il s'agit le plus souvent de relevés du fleuve et de ses abords. L'absence de plans antérieurs à la fin de la période moderne s'explique notamment par le fait que les cours d'eau n'étaient pas une priorité du royaume de France avant le Xvi e siècle (Serna, 1999). Les sources planimétriques comprennent également le cadastre dit «napoléonien » dont les différentes sections sont répertoriées dans des tableaux d'assemblage pour chaque commune. Concernant la zone étudiée, la levée des plans s'est faite dans la première moitié du XIX ${ }^{\mathrm{e}}$ siècle à l'échelle 1/2 500.

L'étude des sources textuelles a été réalisée à partir de trois fenêtres sélectionnées en fonction de leur quantité. Les secteurs concernés sont les communes de Vernou-sur-Brenne et de Noizay situées entre la Loire et la Cisse, ainsi que le cours de la Cisse compris entre Pocé-sur-Cisse et Vouvray (figure 1). L'amplitude chronologique des documents étudiés s'étend du XV ${ }^{\mathrm{e}}$ au XIX ${ }^{\mathrm{e}}$ siècle. Le corpus est principalement composé d'aveux et de devis pour des réparations diverses. La plupart des textes proviennent du Chartrier de Pocé-sur-Cisse ${ }^{3}$ qui rassemble environ 250 cotes.

La quantité de documents portant sur la zone d'étude étant très importante, nous n'avons sélectionné et traité que les informations se rapportant à l'exploitation du sol, aux hameaux et à la Cisse.

\section{Données archéologiques}

L'essentiel des données archéologiques disponibles pour cette étude provient de la base de données Patriarche (Service Régional de l'Archéologie, Centre-Val de Loire) collectées pour la réalisation de l'inventaire archéologique du canton de Vouvray. Le rapport de prospection recense les informations archéologiques selon deux catégories : les sites et les indices de sites (Lauzanne, 1997). Les sites correspondent à des concentrations de mobilier tandis que les indices de sites renvoient à du mobilier isolé provenant généralement du lit de la Loire. Seuls les sites ont été retenus pour cette étude.

3. ADIL, série E. 

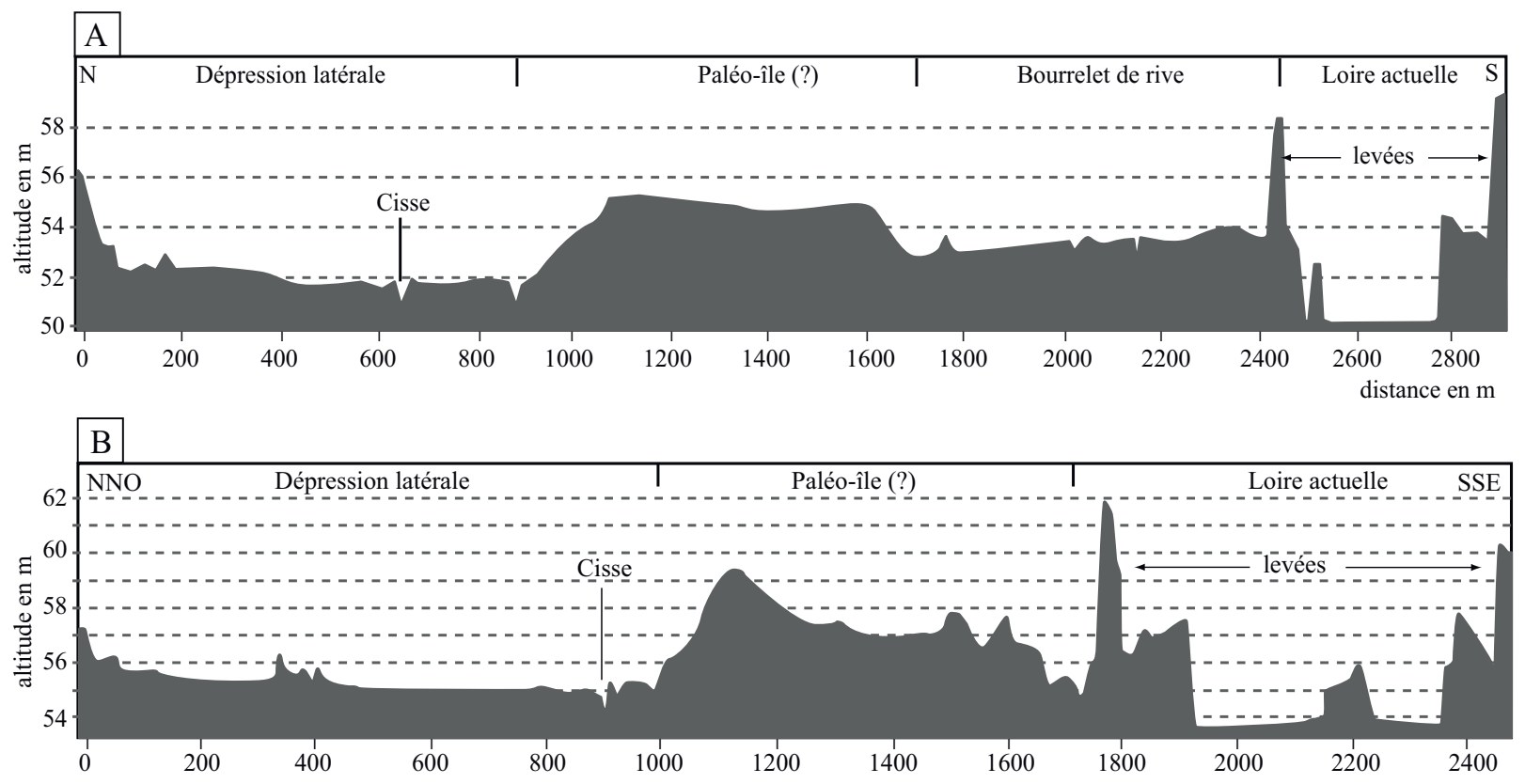

Figure 3 : Exemples de profils transversaux du val de Cisse. A. à la hauteur de Noizay; B. à la hauteur de Pocé sur Cisse. D’après des données LiDAR (DREAL, 2003)

Examples of cross sections of the Cisse Valley: A. At Noizay; B: At Pocé-sur-Cisse. Based on LiDAR data (DREAL, 2003)

\begin{tabular}{|c|c|c|c|}
\hline Cote & Nom & Date & SIG \\
\hline $\mathrm{C} 277$ & Plan des bords de la Loire & XVIII ${ }^{\mathrm{e}}$ siècle & non \\
\hline C113 & Emplacement du moulin de Perreux & $\mathrm{XVIII}^{\mathrm{e}}$ siècle & non \\
\hline $\mathrm{H} 239$ & Plan géométral des Prés Montants & $\mathrm{XVIII}^{\mathrm{e}}$ siècle & oui \\
\hline $\mathrm{H} 289$ & Plan du fief et seigneurie de Négron & XVIII $^{\mathrm{e}}$ siècle & oui \\
\hline II.3.1.1 & Carte de la Loire & XIX siècle & oui \\
\hline II.3.1.24 & Fixation du lit majeur de la Loire & XIX ${ }^{\mathrm{e}}$ siècle & non \\
\hline II.4.17.1 & Plan de la levée de Vernou-sur-Brenne & $\mathrm{XIX}^{\mathrm{e}}$ siècle & non \\
\hline II.4.19.2 & Plan de l'écluse sur la Cisse & - & non \\
\hline 3P2.003.002 & Amboise, section B1 & XIX ${ }^{\mathrm{e}}$ siècle & oui \\
\hline 3P2.156.005 & Montlouis-sur-Loire, Section A & XIX siècle & oui \\
\hline 3P2.156.050 & Montlouis-sur-Loire, cours de la Loire & XIX siècle & oui \\
\hline 3P2.163.002-010 & Nazelles-Négron, sections A à F & XIX siècle & oui \\
\hline 3P2.171.002-009 & Noizay, sections A à E & XIX ${ }^{e}$ siècle & oui \\
\hline 3P2.270.014-017 & Vernou-sur-Brenne, sections H et I & XIX ${ }^{e}$ siècle & oui \\
\hline 3P2.281.012-014 & Vouvray, sections I, K et L & XIX siècle & oui \\
\hline 3P2.185.005-006 & Pocé-sur-Cisse, section D & XIX $X^{\mathrm{e}}$ siècle & oui \\
\hline
\end{tabular}

Tableau 1 : Sources planimétrique

Planimetric data sources

Le corpus est composé de 48 sites sur une surface totale de $23 \mathrm{~km}^{2}$. Ceux-ci ont été regroupés en différents types : habitat, industrie, habitat et industrie et sites dont le type d'occupation n'est pas connu (tableau 2). La répartition des différents types est variable selon les périodes mais le Néolithique, le Moyen Âge et l'époque Moderne sont les mieux renseignées. 


\begin{tabular}{|l|c|c|c|c|c|c|}
\hline & Néolithique & Protohistoire & Gallo-romaine & Médiévale & Moderne & Total \\
\hline Habitat & 2 & - & 3 & 3 & 16 & 24 \\
\hline Industrie & 2 & - & - & 4 & 5 & 11 \\
\hline Mixte & 1 & - & - & - & - & 1 \\
\hline Inconnu & 1 & 8 & 3 & - & - & 12 \\
\hline Total & 6 & 8 & 6 & 7 & 21 & 48 \\
\hline
\end{tabular}

Tableau 2 : Répartition des sites archéologiques par fonction et par période Distribution of archaeological sites by function and by period

Ces données, bien que non exhaustives à l'échelle de la zone étudiée, constituent des repères à la fois chronologiques et relatifs à l'occupation du sol, d'une grande importance pour la compréhension de l'évolution du paysage.

\section{Données géomorphologiques}

La majeure partie des données disponibles est constituée de forages géotechniques compilés dans la Banque du Sous Sol (BSS) du Bureau de Recherches Géologiques et Minières (BRGM), qui n'apportent pas d'information sédimentaire précise sur les dépôts récents (Holocène). Une série de carottages au carottier à percussion et de sondages à la tarière manuelle a été menée afin de répondre à des questionnements spécifiques. Ces informations ont été complétées par des données topographiques à haute résolution.

\section{Méthodes}

L'approche est fondée sur le croisement des données humaines et des données géomorphologiques. D'une part, il s'est agit de réaliser le dépouillement des archives textuelles, cartographiques voire iconographiques, ainsi que des données archéologiques, dans le but de produire une connaissance relativement exhaustive de l'occupation du sol et de sa mise en valeur dans le secteur depuis l'époque médiévale au moins; d'autre part, nous avons produit des données géomorphologiques qui fournissent un premier cadre des dynamiques paysagères à l'Holocène.

L'approche géomorphologique repose sur une étude lithostratigraphique menée à partir de carottages et des sondages à la tarière réalisés dans la dépression latérale et sur le bourrelet de rive (fig. 2A). Des analyses granulométriques ont été réalisées au granulomètre laser afin de caractériser au plus près les dépôts fins. Des données issues du LiDAR (Light Detection and Ranging, système laser aéroporté permettant de construire des modèles numériques d'élévation (MNE) de très haute résolution) et des photographies aériennes, ont permis de mener l'étude détaillée du modelé. Nous proposons ainsi une chronologie de la mise en place du bourrelet de rive et du comblement de la dépression latérale au sein du val de Cisse.

L'ensemble des données produites a été regroupée dans un Système d'Informations Géographiques (SIG) et une Base de données (BDD) (tableau 1). Dès lors, l'approche régressive nous a permis de retrouver un certain nombre d'informations relatives au paysage qui n'étaient pas explicites dans les textes.

\section{RÉsultats}

\section{L'évolution du milieu}

L'étude détaillée des données topographiques et sédimentaires fournit des résultats pour la compréhension de l'évolution morphologique du val de Cisse.

Les données dérivées du LiDAR montrent que le bourrelet de rive est régulièrement coupé par des incisions qui se prolongent dans la dépression latérale. C'est le cas notamment dans la partie aval du val de Cisse (figure 4). Ces incisions dessinent des anomalies topographiques au sein du bourrelet de rive, en rive droite de la Loire actuelle. Il s'agit de formes oblongues bien délimitées, allongées dans le sens amontaval, dont l'altitude est nettement supérieure au fond de la dépression latérale (2 à 3 m) (figures 3 et 4). On les observe également très bien sur les cartes géologiques, pédologiques, sur les photographies aériennes et sur les documents cadastraux du XIX ${ }^{\mathrm{e}}$ siècle (cf. figure 8). Certains de ces bombements topographiques possèdent des traces d'une présence humaine dès le Néolithique, des sites de cette époque ayant été trou- 

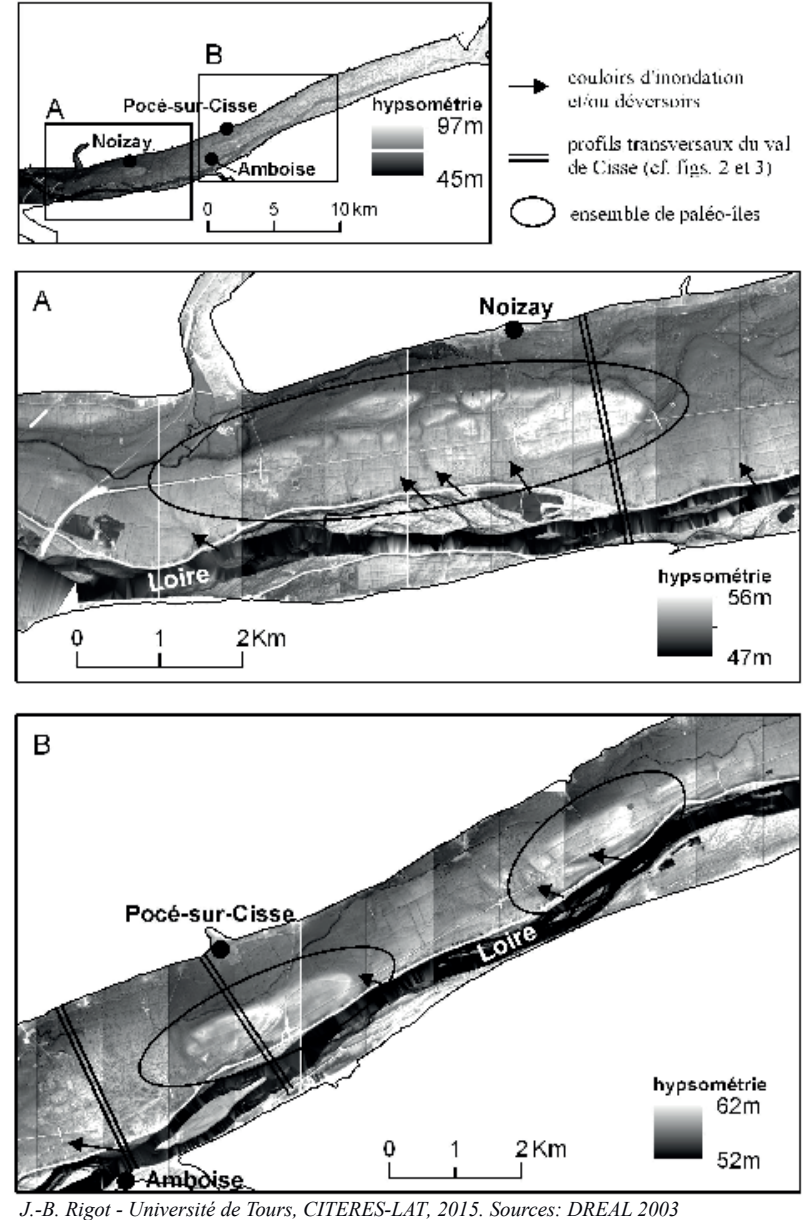

Figure 4 : Anomalies microtopographiques dans le val de Cisse identifiées sur des données LiDAR (DREAL, 2003)

Microtopographic anomalies in the Cisse Valley identified in LiDAR data (DREAL, 2003)

vés en surface. Ces observations mettent en évidence le caractère discontinu du bourrelet de rive dont la signification sera discutée plus loin.

L'analyse des faciès sédimentaires issus des forages de la BSS met en évidence presque systématiquement une couche d'épaisseur variable de sédiments grossiers à la base, sur la roche en place. Il s'agit d'un dépôt de fond de chenal ou du témoignage d'un fonctionnement différent de la Loire.

Au-dessus, les sédiments observés dans les sondages et dans les carottages que nous avons réalisés (ces deniers sont encore en cours de traitement) ainsi que dans les données de la BSS quand cela était précisé, sont, sauf exception, plus fins, sablosilteux, silto-sableux voire silto-argileux.

L'exemple du sondage FAIX 2 (figure 2A, figure 5) réalisé jusqu'à $2 \mathrm{~m}$ de profondeur, témoigne de cette

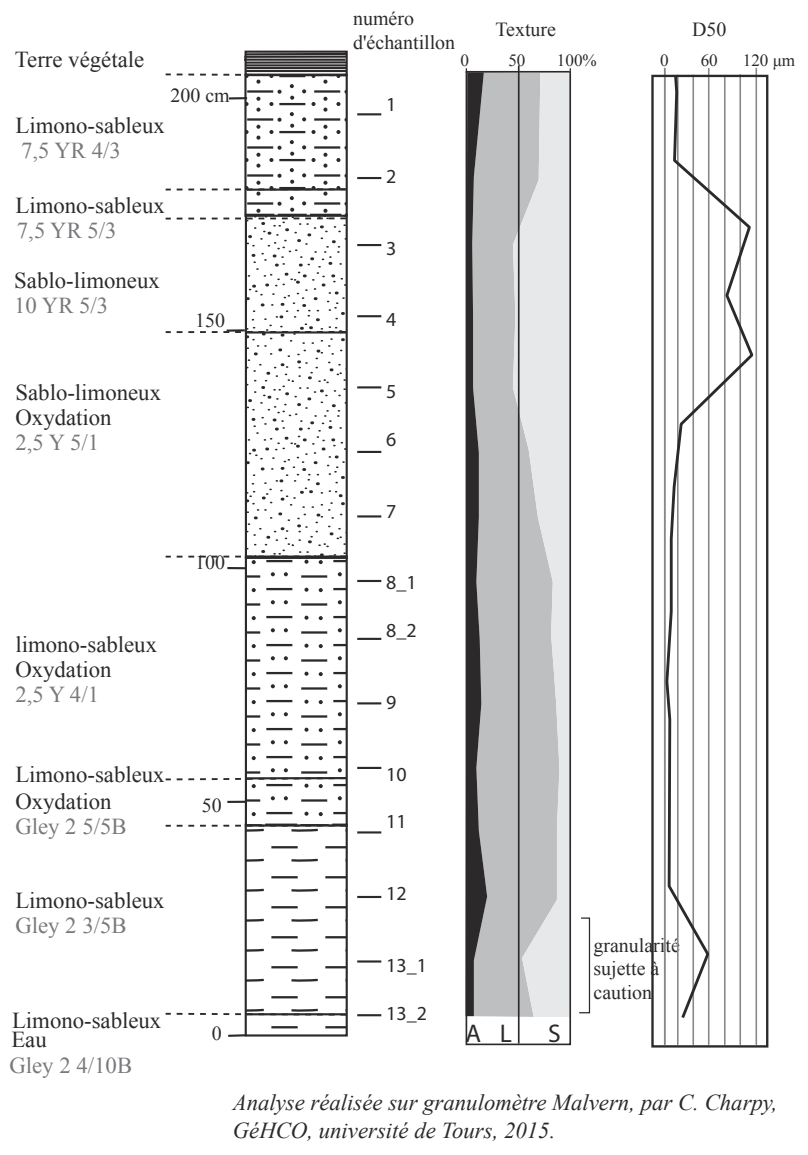

Figure 5 : Carottage Faix2, texture et granularité Faix 2 core, texture and granularity

sédimentation fine. Le dépôt est majoritairement silteux sauf dans une portion de la carotte où un niveau est dominé par les sables. Les analyses granulométriques, qui restent à compléter et à systématiser, montrent qu'il s'agit de dépôts assez mal triés qui correspondent bien à ce que l'on observe généralement dans une plaine d'inondation.

Un carottage (NOY3, figure 2A) a été réalisé sur un bombement topographique appartenant au bourrelet de rive. Il montre un faciès bien différent avec, jusqu'à $2 \mathrm{~m}$ de profondeur un dépôt de sables grossiers et de graviers.

\section{La dynamique du peuplement}

\section{Les premières implantations humaines}

Les données que nous possédons pour le moment sont tirées de S. Lauzanne (1997) et se limitent aux trois communes actuelles de Vouvray, Vernou-surBrenne et Noizay. 
Les 48 sites sont répartis sur ces trois communes qui comprennent respectivement 12, 4 et 32 sites archéologiques toutes périodes confondues (tableau 2). La période gallo-romaine est la plus représentée avec 21 sites alors que seulement 6 sites sont répertoriés pour le Néolithique et le Moyen Âge. Enfin, $50 \%$ du corpus est constitué de sites liés à l'habitat et $33 \%$ de sites dont la fonction n'est pas connue.

Les occupations les plus anciennes sont datées du Néolithique. Les six sites archéologiques s'apparentent, au vu du mobilier lithique retrouvé, à des sites d'habitat et/ou d'artisanat. La plupart d'entre eux sont localisés sur la partie centrale du bourrelet de rive, à mi-distance du fleuve et de son affluent, mais deux sites sont situés sur la rive droite de la Cisse, à proximité immédiate de celle-ci (figure 6).

Pour la période protohistorique, huit sites d'occupation sont connus (figure 6). Le mobilier, principalement composé de céramique non tournée et de silex taillés, n’a pas permis d'identifier la nature de l'occupation. On constate néanmoins que certains sites néolithiques sont réoccupés durant cette période (Les Hauts de Crêne, L'Hommelaye et Les Sables des Petites Vallées). D’autres sites apparaissent et sont tous localisés en bord de Cisse.

L'époque gallo-romaine est représentée par six sites (figure 6). D'après les vestiges, il s'agit de lieux d'habitats. Le fait remarquable est la pérennité de l'occupation depuis le Néolithique et/ou
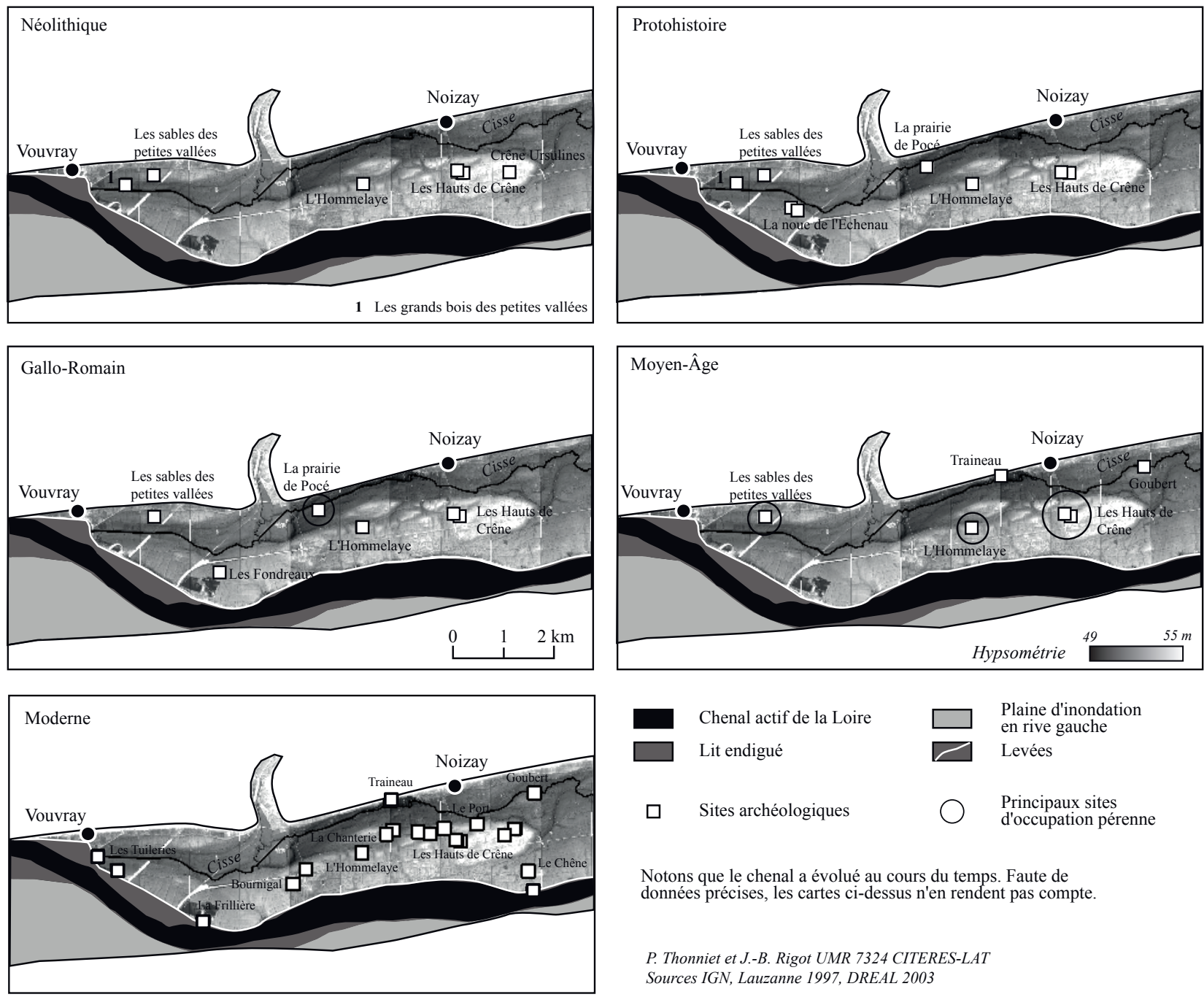

Notons que le chenal a évolué au cours du temps. Faute de données précises, les cartes ci-dessus n'en rendent pas compte.

P. Thonniet et J.-B. Rigot UMR 7324 CITERES-LAT

Sources IGN, Lauzanne 1997, DREAL 2003

Figure 6 : Localisation des sites néolithiques, protohistoriques, gallo-romains, médiévaux et modernes Location of the Neolithic, Protohistoric, Gallo-Roman, Medieval and Modern sites 
la Protohistoire dans trois secteurs : Les Hauts de Crêne, Les Sables des Petites Vallées ainsi que dans la Prairie de Pocé.

Enfin, on n'observe pas de changements quantitatifs pour le Moyen Âge qui est également représenté par un total de six sites (figure 6). Les sources écrites et les vestiges matériels permettent de préciser les implantations : les sites en rive de Cisse correspondent à des moulins hydrauliques, tandis que les autres s'apparentent à de l'habitat, hormis deux sites qui n’ont pas été déterminés (Les Sables des Petites Vallées, daté du haut Moyen Âge, et L'Hommelaye).

\section{Densification de l'occupation et répartition de l'habitat}

Le val de Cisse apparaît comme un espace d'abord rural. Les bourgs, qui se forment durant le Moyen Âge, sont tous situés sur le flan du coteau, en rive droite de la Cisse.

C'est à partir de l'époque Moderne qu'il y a une véritable densification de l'occupation. En effet, en l'espace de quelques siècles le nombre de sites connus a été multiplié par quatre puisqu'ils passent de six à vingt et un (figure 6). Un changement est également visible dans la répartition spatiale des sites puisque c'est uniquement à partir de cette période que se développe une implantation en bord de Loire. Cette migration de l'occupation est probablement due à la mise en place des levées. À l'instar de la période médiévale, les fonctions des sites sont diversifiées et se caractérisent par de l'habitat, de l'industrie et une activité agricole; moulin, tuilerie, granges et fermes sont les témoins les plus fréquents de ces activités.

\section{Les aménagements du val de Cisse}

\section{La mise en place des digues}

L'emplacement et la date de construction des premières digues restent encore mal connus. Elles sont présentes dès l'époque médiévale où elles sont désignées par le terme de turcies. Plutôt qu'un rôle de digue tel qu'on l'entend aujourd'hui, ces premiers aménagements avaient d'abord pour fonction de casser la vague créée par la crue, sans empêcher l'inondation. Les premières turcies ont vraisemblablement été construites dès le viII ${ }^{\mathrm{e}}$ siècle (Serna,
2010 ; Zadora-Rio, 2004 ; Burnouf et Carcaud, 1999; Dion, 1961) mais elles ne sont réellement attestées qu'à partir du XII ${ }^{\mathrm{e}}$ siècle ${ }^{4}$. D'après les travaux récents menés en Loire moyenne, peu de turcies ont été édifiées entre Chouzy-sur-Cisse et Vouvray (Carcaud, 2004).

À partir de la fin du $\mathrm{Xv}^{\mathrm{e}}$ siècle, les turcies sont peu à peu remplacées par des constructions plus élaborées et plus hautes, les levées (Dion, 1961). Ces aménagements, érigés sur tout le cours de la Loire ou presque, corsettent le lit du fleuve et contraignent les eaux à s'écouler dans un couloir étroit (du Bec d'Allier à Nantes, $484 \mathrm{~km}$ sur $487 \mathrm{~km}$ du cours d'eau sont bordés par les levées qui protègent $72 \%$ de la zone inondable [Carcaud, 2004]). La fonction de ces ouvrages est double : favoriser la navigation et maintenir les crues hors des zones inondables afin de protéger les riverains et les cultures. Bien entendu, ce système trop rigide n'a pas pu résister à certains événements extraordinaires et les digues se sont régulièrement rompues, en particulier depuis le $\mathrm{XVII}^{\mathrm{e}}$ siècle. Il a donc fallu réfléchir à la mise en place de "déchargeoirs » afin d'offrir aux crues des zones d'expansion, hors du périmètre des levées. Ce système d'inondation volontaire d'une portion du lit majeur non endigué ne peut concerner que des secteurs faiblement peuplés. Il n'est donc pas surprenant, mais cela apparaît comme une confirmation des observations faites précédemment, que l'un d'eux soit situé dans la partie aval du val de Cisse, sur la commune de Nazelles-Négron, dans un secteur particulièrement peu peuplé (Dion, 1961) (figure 7).

\section{Les moulins}

Cette étude s'est focalisée, pour l'instant, sur les moulins situés entre Pocé-sur-Cisse et Vouvray, dans la partie aval du bassin de la Cisse (figure 7A). Cela correspond à un corpus de quatre moulins sur un total de huit connus dans le val de Cisse. Les sources textuelles et archéologiques renseignent un terminus ante quem ainsi que la fonction de chacun des moulins. Ils sont actuellement encore en élévation et sont tous installés sur des biefs.

Le moulin de Traineau est mentionné dès 1335 dans le cartulaire de l'Archevêché de Tours (Lauzanne, 1997). Il est également évoqué dans les

4. Charte d'Henri II Plantagenêt de 1160 (Carcaud, 2004). 
Figure 7 : Positionnement hypothétique des turcies et du déversoir et localisation des moulins

Hypothetical location of the "turcies" (embankments) and overflow, and the locations of mills

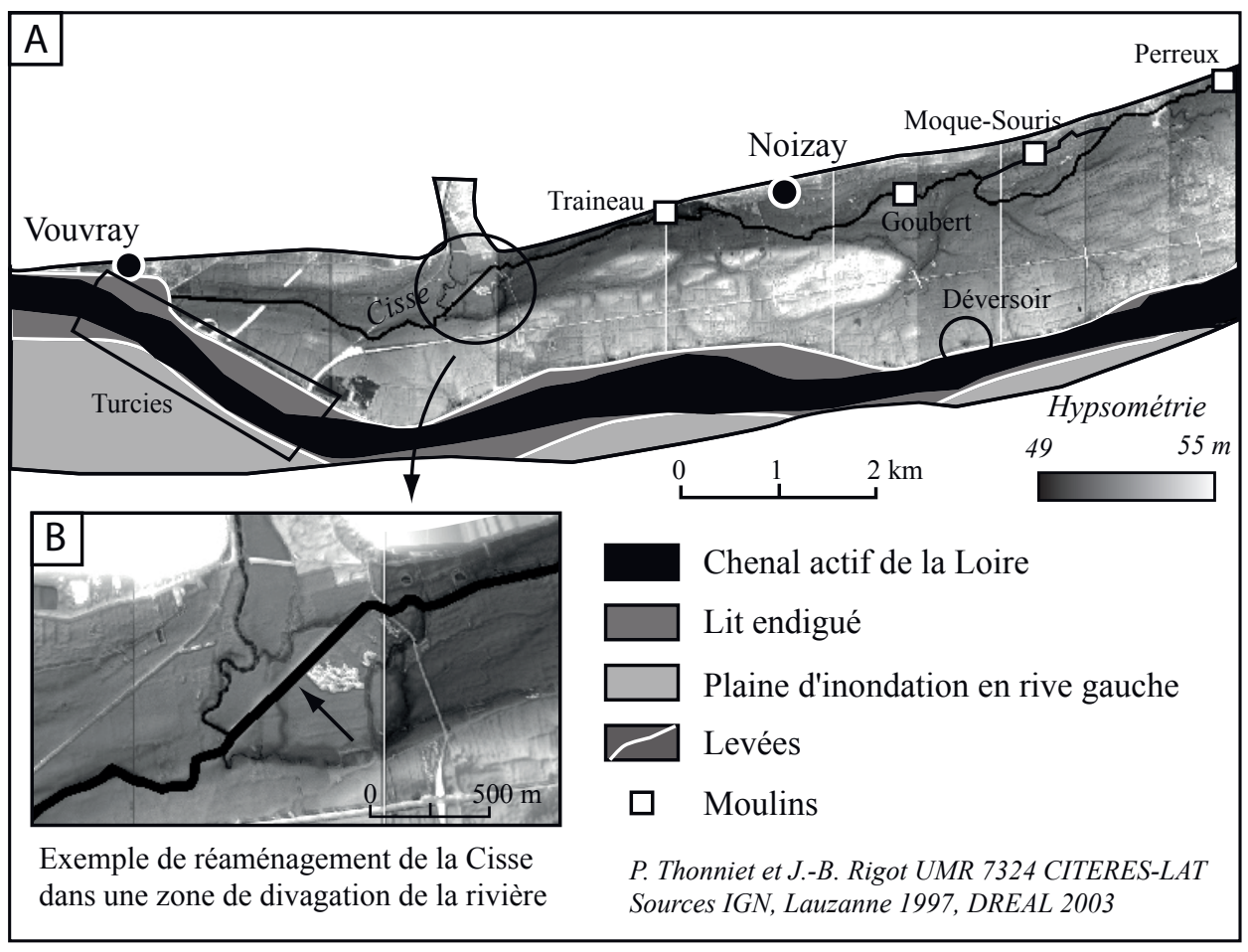

arrêtés de règlement d'eau ${ }^{5}$ du XIX $^{\mathrm{e}}$ siècle. Le moulin de Goubert est cité dans un texte en 1206 (inventaire archéologique de la commune de Vouvray). Par ailleurs, un document des Archives municipales d'Amboise le mentionne en $1511^{6}$. Les arrêtés de règlement d'eau ${ }^{7}$ indiquent que le moulin Goubert était toujours en activité au XIX ${ }^{\mathrm{e}}$ siècle. La mention la plus ancienne connue pour le moulin de Moque-Souris est située sur le cadastre napoléonien du début du XIX ${ }^{\mathrm{e}}$ siècle. Certains documents des Archives nationales ${ }^{8}$ en font également mention après 1830 dans des arrêtés de règlement des eaux. Enfin, le moulin de Perreux figure sur un plan de 1787 conservé aux ADIL ${ }^{9}$. Aucune mention de ce moulin n'est faite dans les arrêtés qui ont été consultés aux Archives nationales.

La présence de ces moulins sur la Cisse confirme l'exploitation de l'énergie hydraulique de la rivière. Afin que celle-ci soit optimale, différents aménagements importants ont été nécessaires : édification de biefs, encore perceptibles dans le paysage; rectification du tracés de la Cisse, dont les cartes sont le témoignage

\footnotetext{
5. $\mathrm{F}^{14} 6142, \mathrm{AN}$.

6. CC124, AMA.

7. $\mathrm{F}^{14} 6142, \mathrm{AN}$.

8. $\mathrm{F}^{14} 6142, \mathrm{AN}$

9. C113, ADIL.
}

évident et que l'on peut observer également sur le terrain (figure $7 \mathrm{~B}$ ); curages ou encore déversoirs ${ }^{10}$ mis en évidence grâce aux plans et aux textes ${ }^{11}$.

\section{Première approche de la morphologie du parcellaire}

L'analyse de la morphologie du parcellaire concerne pour le moment les communes de Noizay et de Vernou-sur-Brenne (figure 1). L'approche régressive a mis en évidence peu de modifications dans la forme du parcellaire depuis le XVII ${ }^{\mathrm{e}}$ siècle.

Les photographies aériennes montrent un parcellaire très laniéré, ce qui transparaît aussi sur le cadastre ancien (figure 8AB). Cependant, l'observation détaillée révèle des formes plus variée et une véritable organisation spatiale des parcelles. Ainsi, les parcelles fortement découpées et de taille réduite, bordées de haies, se situent essentiellement dans la dépression latérale. À l'inverse, des ensembles régulièrement laniérés s'observent

10. $\mathrm{F}^{14} 6142, \mathrm{AN}, \mathrm{Plan}$ "Profil de la rivière après le curage », 1832. Le plan présente différentes sections de la rivière avec l'emplacement de déversoirs pour le bief et l'évolution des nivellements après les curages. 11. C245 et C257, ADIL. Ces documents regroupent des ordonnances et des devis de réparation de ponts sur la Cisse et de curage de la Cisse dans la première moitié du XVIII ${ }^{\mathrm{e}}$ siècle. 

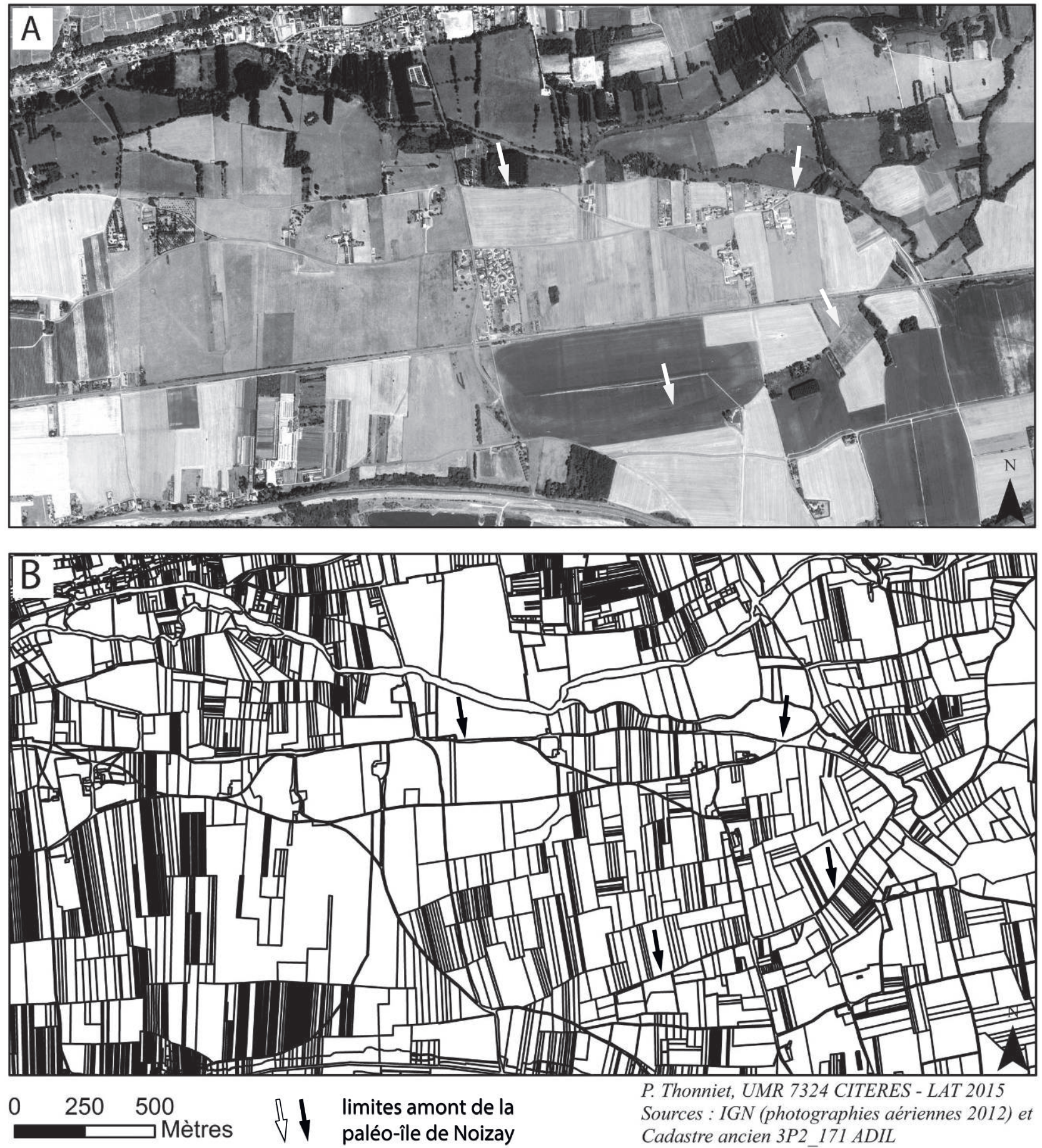

P. Thonniet, UMR 7324 CITERES - LAT 2015

Sources : IGN (photographies aériennes 2012) et Cadastre ancien 3P2_171 ADIL

Figure 8 : Commune de Noizay. A : Photographie aérienne; B : Cadastre ancien vectorisé

Village of Noizay: A. Aerial photograph; B. Old land register, vectorized

plus fréquemment sur le bourrelet de rive. Enfin, les plans témoignent de la mise en valeur des îles qui font l'objet d'un découpage parcellaire (figure 9; pour la localisation de l'île dans l'ensemble du secteur, cf. figure 2).
L'étude des textes se rapportant à la mise en valeur des terres arables, bien qu'encore non exhaustive, a permis de préciser les pratiques agro-pastorales dans le val de Cisse. En confrontant cette analyse avec celle du parcellaire et plus largement celle 
Figure 9 : Parcellaire de l'île du mottage Land use on the Île du Mottage

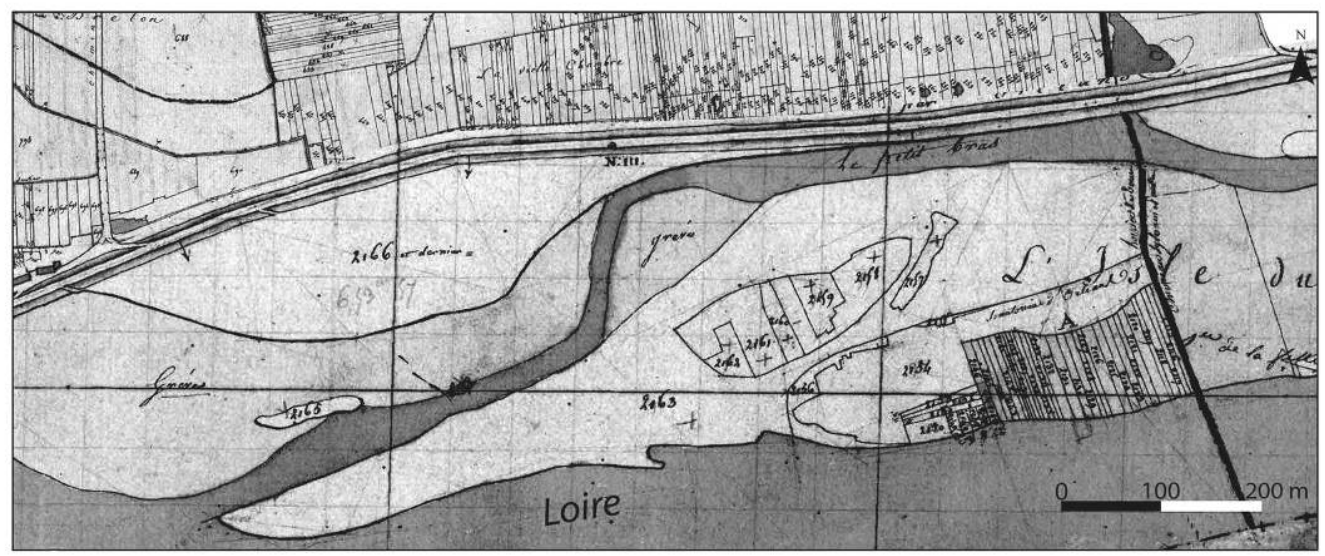

du milieu, deux points retiennent notre attention : d'une part, la mise culture des terres ainsi que l'élevage sont pratiqués dans des zones déterminées par la topographie et la qualité des sols; d'autre part, l'exploitation du sol est relativement variée puisqu'il y a des mentions de terres labourables, de pâtures, de prés ainsi que de saulaies (tableau 3).

\begin{tabular}{|c|c|c|}
\hline Cote & Type de documents & Période \\
\hline E581 & Saisie de biens & 1481 \\
\hline E582 & Inventaire d'aveux & 1570 \\
\hline E584 & Aveux et déclarations & 1488 \\
\hline E593 & Aveux et déclarations & 1713 \\
\hline E598 & Aveux et déclarations & 1651 \\
\hline E601 & Aveux et déclarations & 1572 \\
\hline E605 & Déclaration de fresche & 1462 \\
\hline E615 & Aveux et déclarations & 1641 \\
\hline E616 & Aveux & 1519 \\
\hline E623 & Aveux et déclarations & 1641 \\
\hline
\end{tabular}

Tableau 3 : Sources textuelles

Documentary sources

\section{INTERPRÉTATIONS ET DISCUSSION}

\section{Évolution du milieu}

Les résultats de l'étude fine de la topographie du val de Cisse ont montré une disposition tout à fait similaire à celle observée par Carcaud $(2004,2010)$, en particulier dans le val d'Authion. La genèse de cette morphologie est à rechercher dans les dyna- miques variées ayant caractérisé le cours d'eau depuis le Pléniglaciaire.

Le bourrelet de rive est constitué de plusieurs bombements topographiques bien marqués en particulier en aval d'Amboise (figure 4). Ceux-ci sont recoupés par des incisions latérales qui s'observent bien sur les données issues du LIDAR. Nous interprétons ces formes comme d'anciennes îles séparées par d'étroits chenaux qui auraient progressivement fusionnées pour constituer un bourrelet de rive, après retrait du chenal de la Loire en rive gauche de la vallée. Carcaud (2004, 2010), a également observé des incisions dans le bourrelet de rive du val d'Authion. Elle les interprète comme des couloirs d'inondation. Dans le val de Cisse cette interprétation nous paraît tout à fait plausible, comme le confirment les traitements issus du LIDAR (figure 4). Les incisions, contemporaines des îles, auraient conservé une morphologie déprimée une fois comblée, et auraient été empruntées préférentiellement par les flux lors des débordements de la Loire. Quant à la construction du bourrelet, il s'agit selon Carcaud (2004) de levées naturelles construites sur des barres sableuses. On est là dans un scénario tout à fait proche de l'hypothèse que nous posons pour le val de Cisse.

L'analyse des faciès sédimentaires réalisée pour cette étude, a mis en évidence un dépôt grossier à la base, sur le substrat. Il s'agit de la " grave » ligérienne. Cette nappe alluviale généralisée sur l'ensemble de la plaine, est caractéristique d'une dynamique de cours d'eau en tresse. On peut l'interpréter, après d'autres (Carcaud, 2004), comme les restes de la nappe alluviale de la fin du Weichsélien 
et plus précisément de la phase de tressage du Tardiglaciaire.

Au-dessus de cette nappe s'observent généralement des dépôts plus fins caractérisant une dynamique fluviale bien différente (figure 5). Il s'agit de dépôts issus de suspension graduée ou de suspension uniforme mis en place lors de débordement du chenal actif. Ces dépôts s'observent en particulier dans la dépression latérale. Ils témoignent d'un retrait du chenal actif, ce que confirment d'autres sondages et carottages réalisés dans le secteur.

Sur les bombements topographiques un carottage a montré au moins $2 \mathrm{~m}$ de dépôts grossiers. N'ayant pas pu atteindre une plus grande profondeur, on ne connaît pas l'ensemble de la stratigraphie. Mais ce premier aperçu pourrait attester la présence d'une paléo-îles et confirmer l'hypothèse proposée pour la formation du bourrelet de rive (cf. paragraphes suivants).

Ces observations lithostratigraphiques vont dans le sens du scénario proposé plus haut, avec le retrait du cours de la Loire en un chenal unique en rive sud à la charnière Tardiglaciaire-Holocène. Dès lors la dépression latérale se serait mise en place, marquée par l'édification d'un bourrelet de rive et le colmatage de l'ancien chenal de la Loire avec les dépôts sédimentaires fins décrits plus haut. En effet, en comparant nos résultats avec ceux obtenus sur la Loire en aval du bec d'Allier, dans le cadre du Programme de Recherches sur les Zones Humides (2001), on constate une similarité entre la taille des sédiments et leur appartenance à certaines unités morphologiques de la plaine alluviale. Ainsi, les dépôts provenant de suspension graduée et secondairement de suspension uniforme caractérisent les francs bords, certaines anciennes îles et les bras abandonnés. Notre sondage a été fait en bordure de la dépression latérale, héritière d'un ancien chenal de la Loire comblé. On est donc tout à fait proche des unités géomorphologiques évoquées plus haut. Des bras secondaires ont probablement perduré un temps, mais les dépôts observés pour l'instant témoignent davantage d'un comblement que d'un chenal actif (absence de mécanisme de roulement). Par la suite, la dépression va rassembler les écoulements du coteau et former progressivement la vallée de la Cisse (figure 2).

Ces différentes observations militent en faveur d'un héritage ancien pour ce paysage. Il nous paraît plausible en effet que les îles mises en évidence par le LIDAR se soient façonnées dès la phase de tressage du Tardiglaciaire. Dès lors, nous proposons que la construction du val de Cisse se soit faite en partie par le regroupement progressif de ces îles à la charnière Tardiglaciaire-Holocène, au moment de la rétraction du chenal en rive gauche (figure 2). Il semblerait en effet que cette partie de la plaine alluviale s'exhausse lentement au début de l'Holocène sous l'effet des débordements de la Loire, puis demeure quasiment figée en raison de l'édification des digues corsetant le fleuve. La nappe alluviale observée au-dessus de la grave ligérienne témoigne en tout cas d'une faible capacité de transport liée à une dynamique d'inondation et de comblement. Sa datation confirmera éventuellement ce scénario. Mais la présence de sites Néolithique en surface atteste l'ancienneté du paysage.

\section{L'occupation du val de Cisse}

Les données archéologiques et les sources archivistiques permettent d'appréhender les dynamiques de peuplement du val de Cisse. D'une part, c'est un espace occupé dès le Néolithique. Bien que les datations méritent d'être affinées, on constate qu'il s'agit des occupations les plus anciennes connues à ce jour dans ce type d'espace. Par ailleurs, certains sites sont occupés sur plusieurs périodes, ce qui permet de supposer qu'une occupation permanente et sur la longue durée a pu caractériser cet espace (figure 6). D'autre part, jusqu'à l'époque Moderne, il ne semble pas y avoir d'implantation de l'habitat en des lieux préférentiels, sauf peut-être à l'époque prothistorique où les sites apparaissent localisés majoritairement dans ou au bord de la dépression latérale. Ainsi, l'absence d'une occupation des points hauts est surprenante dans ce secteur. Enfin, le mobilier archéologique met en évidence une différenciation des fonctions entre les sites, au sein d'une même période, mais aussi entre les périodes. Cela traduit une complémentarité et une organisation de l'occupation humaine.

L'implantation des sites semble répondre à des contraintes autres que celles liées au milieu ou à l'aléa puisqu'ils sont établis sans préférence topographique. La répartition de l'habitat telle qu'elle apparaît dans notre étude, témoigne d'une certaine stabilité dans le temps. Le val de Cisse se caractérise par une occu- 
pation essentiellement rurale et dispersée tandis que les bourgs se développent en rive droite de la Cisse, au pied du coteau, probablement le long d'une voie de communication ancienne (Havet, 2015). Ce schéma ne change qu'au moment de l'érection des levées, à la fin du Moyen Âge. La construction de ces digues, censées être insubmersibles, entraîne la création de hameaux sur leur tracé. Certains portent des toponymes associés directement à leur construction comme par exemple « Le Haut Chantier », sur la commune de Limeray (Dion, 1934) tandis que d'autres semblent se développer ex nihilo et profitent de la protection offerte contre les crues. On a donc, à partir de cette période, un accroissement différencié de l'occupation avec une concentration relative de l'habitat au pied du coteau et le long de la levée, et une occupation nettement plus sporadique et isolée dans le reste du val. Cette dynamique n'est pas seulement déterminée par le risque d'inondation mais également par les activités agropastorales qui favorisent la dispersion de l'habitat dans le val, en fonction de la disponibilité des terres agricoles.

L'étude du cadastre ancien et des plans modernes a révélé un découpage parcellaire qui souligne les spécificités de cet espace. En effet, la morphologie du parcellaire, étroitement calquée sur le modelé du val, traduit une organisation spatiale des activités agricoles qui n'est pas surprenante : des secteurs voués aux pâturages, avec la mise en valeur d'espaces humides s'apparentant à un bocage, dans la dépression latérale; des espaces voués à la culture dans les milieux plus secs, sur le bourrelet de rive. Ce constat est confirmé par les textes qui montrent l'adaptation des pratiques agricoles aux contraintes hydrauliques (inondation et hydromorphie).

L'analyse du parcellaire permet également de mettre en évidence des éléments du modelé détectés en particulier grâce aux données LIDAR. Ainsi, la limite nette d'une forme arrondie apparaît clairement dans le parcellaire et témoigne de la présence d'une des paléo-îles identifiées (figure $8 \mathrm{AB}$ et figure 4A; pour sa localisation au sein du secteur d'étude, cf. figure 2). Cela confirme la relation étroite entre la mise en valeur et la morphologie du val sur la longue durée.

Bien qu'il soit difficile de dater précisément le parcellaire, il semblerait que celui-ci soit hérité, au plus tôt, de la période médiévale puisqu'il n'a connu que très peu de modifications.
Les aménagements du cours de la Cisse, quelle qu'en soit la nature, témoignent du rôle crucial de cette rivière pour les populations locales et plus largement, à l'échelle du bassin aval. Bien que la présence de la Loire assure un apport en ressources halieutiques pérenne, il est manifeste que la Cisse, comme la plupart des rivières (Barraud, 2009), a été aménagée pour des usages locaux tels que l'énergie hydraulique (des moulins sont attestés dans les textes dès le XIII ${ }^{\text {e }}$ siècle), les ressources piscicoles, voire le transport (la mention de « Ports » est fréquente). Ces aménagements témoignent d'une rivière " aménagée », "'construite' selon une finalité productive »(Barraud, 2009). La rivière apparaît alors comme une « ressource » territoriale (ibid.). Par ailleurs, son rôle a été fondamental dans le drainage du val au moment de sa mise en valeur agricole. Ce point mérite d'être approfondi, mais les observations faites d'ors et déjà, à partir des textes et des données cartographiques anciennes, montrent que son cours a été canalisé à plusieurs reprises afin de le rendre fonctionnel sur l'ensemble du val et de favoriser le drainage (figure 7).

\section{Conclusion}

Le travail mené dans le val de Cisse apporte un certain nombre d'informations nouvelles concernant sa mise en place et la dynamique du peuplement depuis le Néolithique. Construit au sein de la plaine alluviale, le val de Cisse juxtapose, à l'image des autres vals de Loire moyenne (Carcaud et Garcin, 2001), une dépression latérale, un bourrelet de rive et un chenal actif corseté entre deux hautes digues. Cette morphologie est particulière car elle forme un large atterrissement dans le lit majeur qui a été progressivement occupé par les groupes humains bien qu'exposés directement au risque d'inondation. D'après notre étude, et en la comparant avec les travaux antérieurs (Carcaud, 2004; Castanet, 2008), cette mosaïque fluviale semble être un héritage ancien. Elle résulterait, au passage Tardiglaciaire-Holocène, d'une métamorphose de la Loire, d'un cours en tresses vers un chenal unique. La dépression latérale, occupée aujourd'hui par une rivière partiellement artificialisée (la Cisse) constituerait, en partie au moins, le chenal emprunté par la Loire au Pléniglaciaire, puis par des bras de tressage au Tardiglaciaire. N'ayant pas encore daté 
ces nappes alluviales, nous nous fondons sur les travaux de Carcaud (2004) pour établir notre chronologie. Ainsi, à la charnière Tardiglaciaire-Holocène la Loire se serait progressivement rétractée en un chenal unique en rive gauche et le bourrelet de rive se serait édifié le long du chenal, caractérisé entre autre, par d'anciennes îles et/ou des barres sableuses qui auraient fusionnées. C'est à ce moment qu'une occupation humaine est observée dans le val, avec la présence de sites néolithiques. Ces observations sont en partie corroborées par l'étude de Castanet $(2008,2010)$ dans le val d'Orléans. Celui-ci met en évidence une rétraction du chenal de la Loire dès le début de l'Holocène en raison des héritages Weichséliens qui contraignent l'écoulement. En parallèle il note une baisse de l'activité fluviale jusqu'à $4500 \mathrm{cal}$. BP. Celle-ci reprend à partir de la seconde moitié de l'Holocène, tout comme dans les vals tourangeaux jusqu'à l'Âge du Bronze d'après les observations de Carcaud (2004, 2010).

Il reste encore à systématiser les études sédimentaires et à affiner la chronologie par des datations. Par ailleurs, des analyses polliniques seront effectuées afin de préciser le contexte paléonvironnemental du val. Mais ces premiers résultats sont encourageants pour la compréhension des dynamiques fluviales ligériennes à l'Holocène.

Le val de Cisse apparaît comme un espace particulièrement représentatif du paysage de la Loire moyenne. Il réunit un certain nombre de caractères illustrant la complexité des relations entre les riverains et le fleuve. Caractérisé par sa faible altitude par rapport au chenal actif au sein de la plaine alluviale, elle-même bien incisée dans les calcaires du Tertiaire, le val de Cisse est fortement exposé au risque d'inondation. Cependant, cette situation ne semble pas conditionner directement la répartition de l'occupation du sol. En revanche, la mise en valeur agricole, notamment pour la partie aval du val, semble beaucoup plus dépendante des conditions environnementales. En l'état actuel de la recherche, on a pu mettre en évidence une organisation spatiale qui est assez peu originale mais qui explique bien le paysage actuel. Ainsi, les espaces les plus hydromorphes, correspondant à la dépression latérale, sont consacrés aux activités d'élevage et d'exploitation des prés et des haies, comme en témoigne, en particulier, le cadastre ancien. À l'inverse, les points hauts, en particulier le bourrelet de rive, sont des espaces voués à la culture. On constate une occupation qui, jusqu'à l'époque moderne, ne privilégie pas forcément ces points hauts. C'est même l'inverse qui semble caractériser la période Protohistorique. Nous ne savons pas encore expliquer ce phénomène qui paraît paradoxal dans un tel milieu. Les recherches à venir devraient nous aider à y voir plus clair, en particulier sur l'importance à donner au risque d'inondation dans le secteur. Mais cela confirme déjà les observations faites sur le rôle primordial de la Cisse dans l'organisation spatiale aux différentes époques. On peut supposer qu'une proximité avec le cours d'eau était nécessaire pour les habitants du val, dont la production pouvait alors être facilement transportée par la rivière. Cette hypothèse serait confortée par la mise en évidence de tronçons canalisés de la Cisse, réalisés en vue de rendre la rivière plus fonctionnelle et de limiter les phénomènes d'hydromorphie (figure 7).

Cette occupation dispersée semble se regrouper et s'accroître à l'époque moderne au sein de petits bourgs tous situés en pied de coteaux et le long des digues. En parallèle une occupation sporadique et dispersée caractérise le reste du val. L'essentiel du secteur est presque désert, ce que nous interprétons comme le résultat de la méfiance des populations à l'égard du cours d'eau. La présence d'un déversoir dans le secteur confirmerait l'occupation lâche du val. Pour autant ce dernier est loin d'être inexploité. Cette portion du fleuve apparaît donc de plus en plus comme un espace de ressource agricole pour des exploitants extérieurs au milieu. Ce dernier point paraitt fondamental et sera approfondi dans le cadre des recherches à venir. De la même façon, il faudra préciser la mise en place des digues sur le bourrelet de rive afin de mieux comprendre la chronologie de la mise en valeur de ce territoire. Car s'il paraît faiblement investi par les populations, on a pu montrer que les sites semblent parfois occupés de manière continue depuis l'Antiquité avec une exploitation du sol attestée depuis le Moyen Âge. Il y a donc un éclaircissement à apporter à cette apparente contradiction. L'approfondissement des recherches sur l'ensemble du val, en particulier sa partie amont, devrait nous donner des éléments de réponse à ces différentes questions. 


\section{Remerciements}

Cet article est fondé sur les travaux menés au sein du Laboratoire Archéologie et Territoires (UMR 7324 CITERES), en particulier dans le cadre d'un master puis d'une thèse en cours par P. Thonniet, sous la direction d'E. Lorans et de J.-B. Rigot : La dynamique du paysage et de l'occupation du sol entre Loire et Cisse, de Vouvray à Chouzy-sur-Cisse, pendant l'Holocène.

Les auteurs remercient Elisabeth Lorans qui a accepté de co-encadrer cette thèse ainsi que Samuel Leturcq qui a activement participé à la genèse de ces questionnements sur le Val de Cisse.

Nous remercions également le laboratoire GeoHydrosystèmes Continentaux (GéHCO) de l'université de Tours et en particulier I. Gay-Ovejero sédimentologue, J.-P. Bakyono responsable des analyses sédimentaires, et Charlotte Charpy étudiante en L3 de géologie qui a réalisé les analyses sédimentaires dans le cadre d'un stage à CITERES.

Les auteurs remercient également la Zone Atelier Loire qui a fourni une aide financière pour la réalisation de travaux de terrain.

Enfin, les auteurs remercient les relecteurs pour les remarques constructives. Un grand merci à Céline Rispoli qui a bien voulu corriger la traduction anglaise du résumé et des figures.

\section{Bibliographie}

Alline C., 2005. Les villes antiques du Rhône et le risque fluvial. Gestion des inondations dans les villes romaines. L'exemple de la basse vallée du Rhône, thèse, Université de Provence (Aix-Marseille 1), 3 vol. 348 p., 141 p. et 198 p.

Amoros C., Petrs G.E., 1993. Hydrosystèmes fluviaux, Paris, Masson, coll. «Écologie; 24 », 300 p.

Arnaud-Fassetta G., 2007. L'hydrogéomorphologie fluviale, des hauts bassins montagnards aux plaines côtières : entre géographie des risques, géoarchéologie et géosciences, Dossier d'habilitation à diriger des recherches, université Paris-Diderot (Paris 7), volume 2 : synthèse scientifique, $435 \mathrm{p}$.

Arnaud-Fassetta G., Carcaud N., Castanet C., Salvador P.-G., 2010. Fluviatile palaeoenvironments in archaeological context: Geographical position, methodological approach and global change - Hydrological risk issues, Quaternary International, Volume 216, Issues 1-2, 1, p. 93-117.

Arnaud-Fassetta G., Carcaud N. (dir.), 2015. La géoarchéologie française au $\mathrm{XXI}^{e}$ siècle, CNRS Alpha, $620 \mathrm{p}$.

Arthuis R., Monteil M. (dir.), 2015. Archéologie de la BasseLoire, Rennes, PUR, coll. «Archéologie et culture », 194 p.
BARraud R., 2009. La rivière aménagée et le moulin à eau. Un héritage en déshérence ? Trajectoires, modèles et projets de paysages. Exemples des vallées sud-armoricaines. Bulletin de l'Association de géographes Français, 86-1, p. 32-45.

Bazin P., Gautier E., 1996. Un espace de liberté pour la Loire et l'Allier : de la détermination géomorphologique à la gestion, Revue de Géographie de Lyon, 71/4, p. 377-386.

Bravard J.-P., 2004. Le risque d'inondation dans le bassin du Haut Rhône : quelques concepts revisités dans une perspective géohistorique, in Burnouf J., Leveau P. (éd.), Fleuves et marais, une histoire au croisement de la nature et de la culture, Paris, CTHS, p. 397-408.

Bravard J.-P., Burnouf J., Vérot A., 1989. Géomorphologie et archéologie dans la région lyonnaise. Questions et réponses d'un dialogue interdisciplinaire, Bull. Soc. Préhist. Fr., 10-12, p. 429-440.

Bravard J.-P., Jacquet C., Amoros C., Vérot A., Burnouf J., 1987. Le site gallo-romain de la Place Bellecour à Lyon, reconstitution interdisciplinaire du paléoenvironnement, in La ville et le flewve, Congrès du CTHS de Lyon, 1986.

Bravard J.-P., Petit F., 1997. Les cours d'eau, dynamique du système fluvial, Paris, Armand Colin, coll. «U », 222 p.

Burnouf J., Carcaud N., 1999. Le Val de Loire en Anjou Touraine : un cours forcé par les sociétés riveraines, Médiévales, 36, p. 17-29.

Burnouf J., Carcaud N., 2000. L'homme et les vallées : les vals de Loire de Tours à Angers, Annales de Bretagne et des pays de l'Ouest, 107, 1, p. 7-22.

Burnouf, J., Carcaud, N., Cubizolle, H., Garcin, M., Trément, F., Visset, L., 2003a. Interactions sociétés/milieux dans le bassin versant de la Loire depuis le Tardiglaciaire jusqu'à la période industrielle, in Muxart T. (éd.), Des milieux et des hommes : fragments d'histoires croisées, Paris, Elsevier, p. 65-77.

Burnouf J., Carcaud N., Garcin M., Giot D., Galinié H., Rodier X., Marlet O., Blin C., Taberly C., 2003b. Fluvial metamorphosis of the River Loire during the Holocene and the role of natural and anthropogenic factors: a case study from the area of Tours, France, in Proceedings of the Alluvial Archeology of NW Europe and Mediterranean, 18-19 décembre 2000, Leeds, Balkema, p. 163-171.

Carcaud N., 2004. D'espace et de temps: un itinéraire de recherche et d'enseignement sur les anthroposystèmes fluviaux, Mémoire d'HDR, Université d'Angers, 2 volumes.

Carcaud N., Garcin M., Visset L., Burnouf J., 2002. Nouvelle lecture de l'évolution des paysages fluviaux à l'Holocène dans le bassin de la Loire moyenne, in Bravard J.-P., Magny M. (éd.), Les fleuves ont une histoire, paléo-environnement des rivières et des lacs français depuis 15000 ans, Paris, Errance, p. 71-84.

Collectif, 2001. Programme National de Recherche sur les Zones Humides, rapport final des travaux, ministère de l'Environnement, Agence de l'eau, 220 p.

Castanet C., 2008. La Loire en val d'Orléans. Dynamiques fluviales et socio-environnementales durant les derniers 30000 ans: de l'hydrosystème à l'anthroposystème, thèse de l'université Paris 1 Panthéon Sorbonne, 485 p. 
Dion R., 1934. Le Val de Loire. Étude de géographie régionale, Tours, Arrault, $752 \mathrm{p}$.

Dion R., 1961. Histoire des levées de la Loire, Paris, 316 p.

Grivel S., 2008. La Loire des îles, du Bec d'Allier à Gien : rythme d'évolution et enjeu de gestion, thèse de l'université Paris VIII, $517 \mathrm{p}$.

Havet M., 2015. Les voies antiques entre Orléans et Tours. Mémoire de master 2 d'archéologie, Tours, Université François-Rabelais.

Knighton D., 1984. Fluvial forms and processes. Edward Arnold, London, 218 p.

Lauzanne S., 1997. Inventaire archéologique, Canton de Vouvray.

Leturce S., 2004. La vie rurale en France au Moyen âge: $X^{e}-$ $X V^{e}$ siècle, Paris, Armand Colin, coll. " Cursus », 180 p.

Morhange C., Provansal M. (éd.), 2007. Géoarchéologie. Numéro spécial de Géomorphologie : relief, processus, environnement, $104 \mathrm{p}$.

Morin E., 2011. Évolution morphosédimentaire de la vallée de la Choisille (sud-ouest du Bassin parisien, France) depuis le Weichsélien. Spécificité de l'impact climatique et anthropique en Europe du Nord-Ouest, thèse de doctorat, Université François-Rabelais de Tours, France, 492 p.

Peтrт C. (dir.), 2005. Occupation et gestion des plaines alluviales dans le Nord de la France de l'âge du Fer à l'époque galloromaine, Actes de la table-ronde de Molesme, 17-18 septembre 1999, Besançon, Presses universitaires de FrancheComté, 214 p.
SAlvador P.-G., 2005. Géomorphologie et géoarchéologie des plaines alluviales (piémont alpin et nord de la France). Dossier d'habilitation à diriger des recherches, texte de synthèse, université des Sciences et Technologies de Lille 1, 298 p.

Schumm S. A., 1977. The fluvial system, New York, Wiley, 338 p.

SERna V., 1999. Le fleuve de papier. Visites de rivières et cartographies de fleuve (XIII- ${ }^{\mathrm{e}} \mathrm{XVII}{ }^{\mathrm{e}}$ siècles), Médiévales, 36, p. 31-41.

Thonniet P., 2013. La dynamique du paysage et de l'occupation du sol entre Loire et Cisse, de Vouvray à Pocé-sur-Cisse, mémoire de master 1 d'Archéologie, Université de Tours, $53 \mathrm{p}$.

Thonniet P., 2014. La dynamique du paysage et de l'occupation du sol entre Loire et Cisse, de Vouvray à Pocé-sur-Cisse, mémoire de master 2 d'Archéologie, Université de Tours, $164 \mathrm{p}$.

Van Der Leeuw S., Favory F., Fiches J.-L., 2003. Archéologie et systèmes socio-environnementaux. Etudes multiscalaires sur la vallée du Rhône dans le programme ARCHAEOMEDES, CRA-Monographie, $\mathrm{n}^{\circ}$ 27, $408 \mathrm{p}$.

Zadora-Rio E., 2004. Aménagements hydrauliques et inférences socio-politiques : études de cas au Moyen Âge, in Burnouf J. et Leveau Ph. (dir.), 2004. Flewves et marais, une histoire au croisement de la nature et de la culture, Paris, CTHS, p. 387-393. 